\title{
La dimension culturelle et identitaire dans l'ethnomusicologie actuelle du domaine français
}

Dimensions of Culture and identity in current French ethnomusicology

\author{
Luc Charles-Dominique
}

\section{(2) OpenEdition}

\section{Journals}

Édition électronique

URL : http://journals.openedition.org/ethnomusicologie/1257

ISSN : 2235-7688

Éditeur

ADEM - Ateliers d'ethnomusicologie

Édition imprimée

Date de publication : 1 décembre 1996

Pagination : 275-288

ISBN : 978-2-8257-0559-9

ISSN : 1662-372X

\section{Référence électronique}

Luc Charles-Dominique, "La dimension culturelle et identitaire dans l'ethnomusicologie actuelle du

domaine français », Cahiers d'ethnomusicologie [En ligne], 9 | 1996, mis en ligne le 05 janvier 2012, consulté le 01 mai 2019. URL : http://journals.openedition.org/ethnomusicologie/1257

Ce document a été généré automatiquement le 1 mai 2019.

Tous droits réservés 


\title{
La dimension culturelle et identitaire dans l'ethnomusicologie actuelle du domaine français
}

\author{
Dimensions of Culture and identity in current French ethnomusicology
}

\author{
Luc Charles-Dominique
}

1 Il n'est pas de recherche ethnomusicologique actuelle et de valorisation de cette recherche - par l'écrit, l'image ou le son - qui n'attestent de l'extrême péril dans lequel se trouvent les phénomènes étudiés et les contextes qui les ont produits. Et encore, dans ces cas-là, parle-t-on de survivances, malgré leur fragilité et l'imminence de leur disparition. Car combien d'ethnomusicologues, et plus largement d'ethnologues, ont-ils déjà constaté la perte irrémédiable de pratiques sociales, communautaires, rituelles, religieuses, dans des sociétés aussi diverses que les mélanésiennes, indiennes, inuits, africaines, asiatiques, européennes... ? Du génocide physique et culturel (par exemple les Tibétains, les Indiens du Canada, d'Amérique du Nord, d'Amérique andine et d'Amazonie, etc.) à l'acculturation provoquée par la colonisation, l'évangélisation et l'islamisation forcées, l'industrialisation, l'urbanisation, les télécommunications... il n'est pas de société épargnée, et ceci quel que soit le degré, très relatif, de son « isolement ». Lorsque Hugo Zemp, en 1978, écrit que «les musiques les plus populaires d'aujourd'hui » chez les 200000 habitants des Iles Salomon (pourtant, à cette date, Mélanésiens à $96 \%$ ) sont « les chorals protestants, la musique néo-polynésienne avec guitare et ukulélé et la musique pop anglo-saxonne » (Zemp 1978), l'on mesure immédiatement l'ampleur et l'universalité $\mathrm{du}$ phénomène. Et ce ne sont pas des décisions politiques étatiques autoritaires (limitation du taux de pénétration des musiques « étrangères » - c'est-à-dire occidentales -, élaboration de genres musicaux «nationaux », etc.) qui sont de nature à renverser ces processus. On est même en droit de se demander dans quelle mesure elles ne contribuent pas à accélérer les phénomènes d'hybridation musicale et donc la disparition des pratiques musicales en question.

2 Cet état de fait, assez récent, dont on peut faire remonter dans certains cas l'origine à quelques décennies tout au plus (notamment dans certaines sociétés extra-européennes) 
s'est, bien entendu, considérablement accéléré depuis la fin de la seconde guerre mondiale. Si bien que l'ethnomusicologie, discipline récente dont Charles Boilès et JeanJacques Nattiez font remarquer que son histoire est presque synchronique (Boilès, Nattiez 1977 : 27), et dont l'objet a toujours été majoritairement extra-européen, subit cette mutation évidemment très déstabilisante. D'autant que ce déplacement des fonctions et des situations des faits musicaux étudiés par l'ethnomusicologie s'accompagne de déplacements de populations, de gigantesques brassages ethniques, culturels, non seulement dans les mégapoles, mais également dans tout le tissu urbain des grands pays industrialisés ou en cours d'industrialisation. En ce qui concerne les ethnomusicologues européens, il n'est plus nécessaire aujourd'hui de choisir des "terrains » forcément lointains : le déplacement partiel de certaines communautés dans des pays occidentaux fait que l'on peut assister aux Nouvel-An chinois à Paris, iranien à Toulouse... Certains des plus grands maîtres proche- ou moyen-orientaux ou bien asiatiques résident aujourd'hui dans les grandes capitales occidentales, dans lesquelles ils se produisent, enseignent, s'associent à des musiciens autochtones ou issus d'autres communautés immigrées... Cette évolution n'a d'ailleurs pas que des effets négatifs pour l'avancée de l'ethnomusicologie: combien notre connaissance des musiques vietnamiennes s'est-elle enrichie depuis la venue en France de Trân Van Khê et Trân Quang Haï !

En résumé, les notions même de déclin, de disparition, de bouleversement, de transformation radicale et irréversible, d'acculturation ou d'hybridation, de mise en situation d'interculturalité, semblent désormais immanentes aux sociétés dites encore "traditionnelles ", quelles qu'elles soient. Cependant, si cette expansion des modèles culturels occidentaux à l'ensemble des peuples est aujourd'hui une réalité unanimement observée, je voudrais essayer de montrer en quoi la situation est particulière en Europe occidentale, et plus spécialement en France, puisque nous sommes au cœur du problème et avons subi, et souvent bien avant les autres, les affres de "l'occidentalisation ». De plus, l'approche des musiques traditionnelles en France, depuis le début des années 1970, est très différente de celle de l'ethnomusicologie en général, et je partage totalement l'avis de Jean-Pierre Estival lorsqu'il écrit que « les musiques traditionnelles ne relèvent pas d'un concept ou d'une catégorie scientifique, mais représentent bien, dans la France d'aujourd'hui, une catégorie culturelle» (Estival 1995: 146). Au moyen de quelques références à l'action du Conservatoire Occitan de Toulouse, Centre des musiques et danses traditionnelles en Midi-Pyrénées, association à laquelle je collabore depuis 1977, je voudrais illustrer cette antériorité de l'action culturelle sur l'approche scientifique. Plus particulièrement, je voudrais montrer en quoi les facteurs historiques, sociaux, économiques, politiques et idéologiques, ainsi que la disparition quasi générale des contextes de la musique traditionnelle en France, ont influencé nos choix et notre action, ont contribué à nous engager activement dans le mouvement de renouveau des musiques traditionnelles, et donc à devenir des acteurs culturels, puis plus tard des ethnomusicologues, nous ont orienté vers une "ethnomusicologie du présent», une ethnomusicologie "de l'utile», une ethnomusicologie prospective et « militante »'. Avec, en arrière-plan, les débats fondamentaux sur la revendication régionaliste et identitaire, et sur la notion de tradition, plus exactement sur la survivance ou non des pratiques musicales et chorégraphiques traditionnelles.

4 Si tant est qu'il faille attribuer un point de départ au mouvement de renouveau des musiques traditionnelles, encore appelé revival, comment ne pas prendre en considération le mouvement folklorique, apparu en France dans certains endroits (ceux 
du thermalisme pyrénéen par exemple) avec une extraordinaire précocité (vers 1890)? Bien qu'il ne s'agisse pas ici d'en proposer une quelconque exégèse, force est de constater qu'il présente à la fois les traits d'un courant puissamment organisé, implanté, allant même jusqu'à proposer une nouvelle forme de sociabilité, et ceux d'un mouvement fortement idéologisé, dont le moteur est une volonté affichée de sauvegarde (conservatisme) ou de réhabilitation de pratiques musicales et chorégraphiques censées caractériser la ruralité, valeur refuge contre un ensemble de valeurs émanant de l'urbanité, notamment de la classe ouvrière. Le folklore, en France tout au moins, est donc bien un engagement culturel, régionaliste, un choix idéologique, même si cette construction identitaire prendra une tournure différente des courants régionalistes postérieurs aux événements de mai 1968.

Fig. 1 : Danseurs de rondeau. 1969. Enquêtes des Ballets Occitans à Mirepoix du Gers. Fonds documentaire Conservatoire Occitan

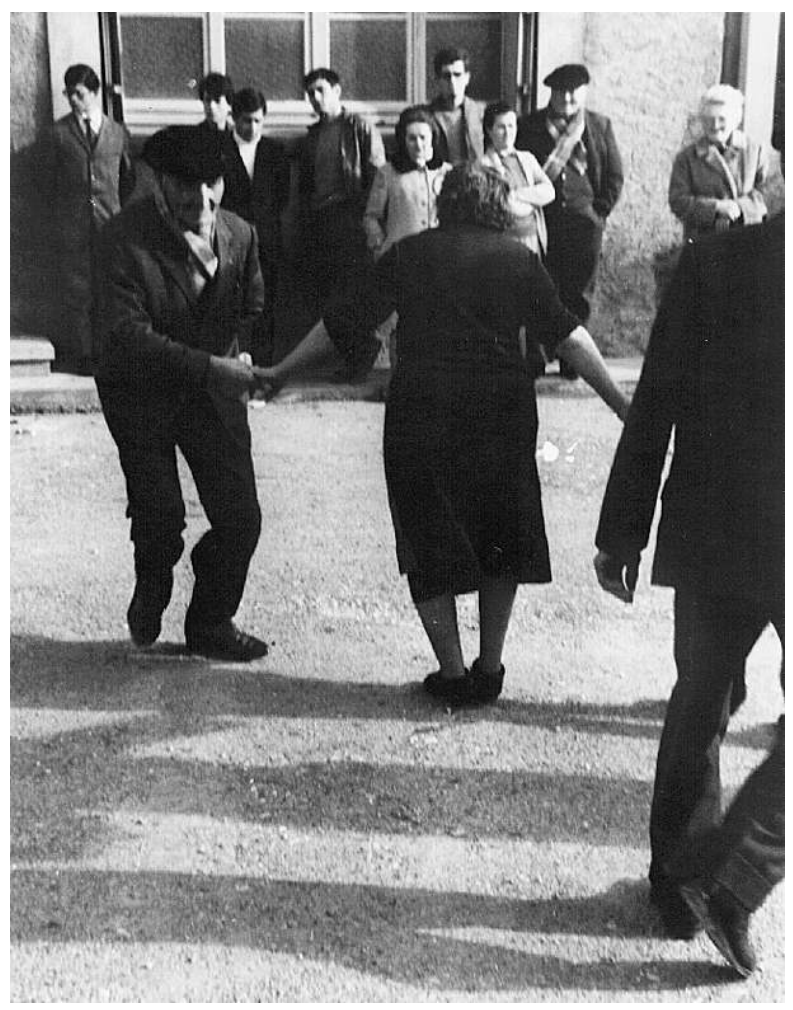

Dans de nombreuses régions, notamment l'Ouest, le Sud-Ouest et le Sud-Est (peut-être l'identité linguistique y est-elle un facteur déterminant, encore que, de ce point de vue-là, d'autres régions comme le Nord ou l'Alsace seraient à prendre en considération), on recense de véritables courants folkloriques institutionnalisés: Cercles celtiques, fédérations folkloriques provençales, etc. Au début des années 1960, dans deux régions bien différentes, le Poitou et la région toulousaine, deux troupes folkloriques vont jouer un rôle déterminant dans la genèse du revival. Il s'agit respectivement du Ballet Poitevin ( Les Pibolous) et des Ballets Occitans (les seconds sont antérieurs aux premiers). Ces troupes sont les premières en France à proposer une adaptation scénique de la thématique "des travaux et des jours ", c'est-à-dire de tout un ensemble de savoir-faire, fêtes, rites et cérémonies, le tout selon une trame diachronique du type « $d u$ berceau à la tombe " (pour paraphraser Arnold Van Gennep) ou plus idéologique du type «hier pour demain ». Ces spectacles sont alimentés par de nombreuses collectes. Dans le cas des 
Ballets Occitans, en 1963 et 1964: collectes en Rouergue, Montagne Noire et BasLanguedoc; de 1965 à 1967 : Montagne Noire (enquêtes sur l'aire de jeu de la cornemuse languedocienne bodega); en 1968 : Lomagne et Astarac (Gascogne), Ariège, Rouergue. On s'aperçoit que, déjà dans les années 1960, une certaine forme de régionalisme est associée à la collecte des musiques et des danses traditionnelles (je dirai même qu'il est conditionné par elle). Ces collectes folkloriques sont parmi les premières du mouvement de collecte entrepris par le secteur associatif, mouvement qui sera prolongé par le revival. Il est d'ailleurs étonnant de constater que, si cette nouvelle conception "Arts et Traditions Populaires » du folklore doit beaucoup à Georges Henri Rivière, les grandes enquêtes ethnomusicologiques du domaine français réalisées par Claudie Marcel-Dubois ou sous sa direction depuis les années 1950 jusqu'à la fin des années 1970 (Pyrénées, Aubrac, Landes...) resteront longtemps ignorées à la fois de ces folkloristes et plus tard des premiers acteurs du revival ${ }^{2}$.

6 En Poitou comme dans la région toulousaine, le succès des Ballets Poitevins et Occitans est considérable, posant bientôt la question de l'importance des moyens à mettre en œuvre pour mener à bien des campagnes systématiques de collecte. En Poitou naîtra en 1968 l'Union Pour la Culture Populaire en Poitou-Charentes-Vendée (UPCP), fédération d'associations de culture populaire, dont certaines vont se consacrer plus spécifiquement à la musique et à la danse traditionnelles poitevines. A Toulouse, à la fin 1970, sera créé le Conservatoire Occitan, association chargée alors de poursuivre la recherche des Ballets Occitans, de travailler au renouvellement de la matière musicale et chorégraphique de leur spectacle, mais aussi d'œuvrer à la formation d'éventuelles futures recrues, et bientôt d'entamer la gigantesque tâche de la réhabilitation d'une partie de l' instrumentarium traditionnel gascon et languedocien, notamment tout ce qui concerne les instruments de lutherie tournée (hautbois, cornemuses) et qui constitue la spécialité actuelle de l'atelier de facture instrumentale du Conservatoire Occitan. Le cas n'est pas totalement isolé : combien d'acteurs importants du revival sont-ils directement issus du mouvement folklorique (je pense au groupe La Chavannée de Montbel en Bourbonnais, ou à de fortes et marquantes individualités provençales comme Patrice Conte - groupe musical Mont Joïa - ou Miquèu Montanaro).

Cependant, la création de ces grandes associations - structures professionnelles - n'est pas seulement guidée par des préoccupations utilitaires. Elle s'inscrit également dans un large courant qui marque en profondeur la décennie 1970 et qui est celui, entre autres, d'un rejet de la culture "dominante », d'une lutte pour la reconnaissance des cultures «dominées", du respect $d u$ "droit à la différence", d'une forte revendication régionaliste et écologiste. Cet engagement culturel et politique, dans de nombreuses régions, notamment les régions périphériques de l'Ouest, du Sud-Ouest et du Sud-Est, se traduira par la volonté marquée de réhabiliter les parlers régionaux ainsi que le patrimoine musical et chorégraphique traditionnel. L'adéquation est forte entre ces deux centres d'intérêts, ces divers idiomes contribuant à élaborer une langue commune, une koinè culturelle constitutive de l'identité. Sans doute, le recours appuyé des jeunes Américains anti-militaristes, en quête d'une nouvelle forme de sociabilité, aux sources de leur propre musique - le protest-song étant directement dérivé du folk-song - est-il à prendre en considération dans le mouvement de renouveau des musiques traditionnelles en Europe occidentale et plus spécifiquement en France. Quoi qu'il en soit, le revival s'accroissant de manière inattendue, la tâche multiple qui était assignée à certaines associations se restreint pour se spécialiser dans le domaine prioritaire des musiques et 
des danses traditionnelles. Il en est ainsi du Conservatoire Occitan, qui abandonne petit à petit dans le courant des années 1970 sa vocation d'association «d'Arts et Traditions Populaires $»^{3}$.

Fig. 2 : De gauche à droite: MM. Michel Biau, Léon Larose (hautbois) et Sabatier (tambour), tous musiciens languedociens, en 1971, au Conservatoire Occitan, lors d'une "veillée de ménétriers ». Le hautbois est celui des Joutes nautiques de Sète. Collection Conservatoire Occitan.

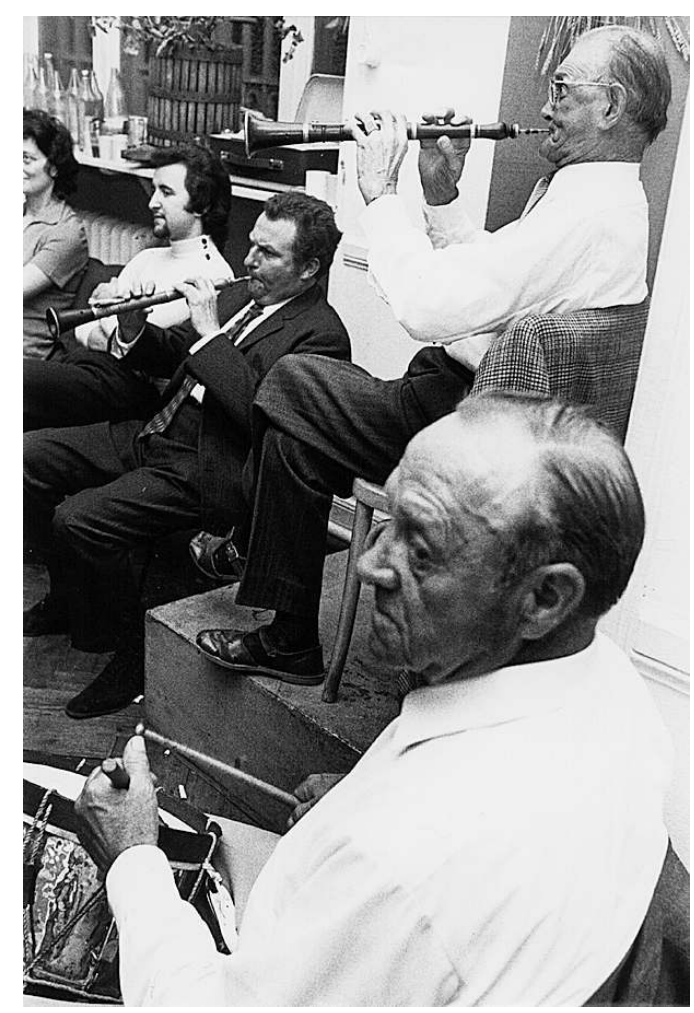

La collecte participant en premier ressort au processus de validation de toute entreprise dans le domaine des musiques et danses traditionnelles, dans les régions concernées par la revendication identitaire on constate rapidement une identification de l'entité régionale telle qu'elle est posée dans le discours régionaliste au «terrain » du collecteur (qui, à cette époque, n'est généralement pas encore apprenti-ethnomusicologue). Plus que jamais ce « terrain » est doté d'un statut « territorial ». Alors que l'on voit émerger à la fin des années 1970 et au début des années 1980 une quantité d'entreprises de collectes très localisées, les champs explorés apparaissent le plus souvent comme des zones d'exclusion. En même temps que le «terrain » légitime l'existence même du collecteur, éventuellement l'action du musicien traditionnel, il est constitutif de leur pouvoir, de leur autorité. Et ceci d'autant plus que l'on peut encore y déceler quelques vestiges instrumentaux ou chorégraphiques pertinents. Car, de quelque manière que l'on se situe dans le débat concernant la rupture ou au contraire les survivances dans la perpétuation des pratiques traditionnelles, il ne fait de doute pour personne que les musiciens traditionnels recensés et enregistrés dans les années 1970 constituent la dernière génération d'une certaine forme de pratique musicale. Ce n'est pas là pur fantasme, un lieu commun à tout folkloriste qui se respecte (le thème de l'urgence de la collecte est déjà présent au xix ${ }^{e}$ siècle, chez les correspondants de l'enquête Ampère-Fortoul). En effet, si les musiciens ou danseurs des bals traditionnels des années 1920-1930 n'ont 
qu'une filiation assez lointaine avec leurs ancêtres, leur pratique musicale ou chorégraphique relève de processus qui n'ont plus cours aujourd'hui.

Cependant, malgré la conscience assez largement répandue de l'urgence, les collecteurs des années 1970 n'ont généralement pour eux que leur bonne volonté. D'une part les conditions de l'enquête (matériel d'enquête compris) sont le plus souvent désastreuses. D'autre part, il manque à la plupart d'entre eux une formation scientifique. Les premières collectes font apparaître, dans l'ensemble, une absence totale de méthodologie : les faits musicaux ne sont pas abordés dans leur globalité, ne sont pas étudiés dans leur essence, leur fonction sociale, rituelle ou religieuse. Les musiciens ne sont pas mieux considérés : ni leur origine, ni leur formation, ni leur itinéraire personnel, ni leurs expériences diverses ne transparaissent dans les bandes d'entretiens de la fin de la décennie 1970 (je me réfère ici au fonds documentaire audiovisuel du Conservatoire Occitan). Seuls comptent alors les répertoires musicaux ou de danses et, bien entendu, les instruments de musique lorsqu'ils suscitent chez le collecteur un intérêt particulier. Dans les diverses provinces alentour de Toulouse, on ne peut pas vraiment parler d'ethnomusicologie à cette époque-là. Il existe d'ailleurs un tabou vivace à l'égard des universitaires en général, l'ethnomusicologue étant perçu comme un personnage « de l'extérieur », en provenance d'un «ailleurs » intellectuel mais aussi géographique et dont les intentions ne sont pas forcément louables (on lui fait souvent grief de « confiscation » des patrimoines musicaux et chorégraphiques traditionnels). De plus, le poids de l'institution universitaire ou muséographique à laquelle il appartient ne prêche pas en sa faveur. Le revival français est essentiellement associatif et la jonction avec le secteur institutionnel n'interviendra que plusieurs années plus tard.

Fort heureusement, les années 1980, en même temps qu'elles voient l'enthousiasme des origines se ternir quelque peu, connaissent la maturation du revival français, avec une reconnaissance ministérielle déterminante dans l'organisation de ce mouvement, dans son développement, dans la prise en compte de l'enseignement des musiques traditionnelles par les institutions musicales (écoles de musique, conservatoires). Quelques événements de ces quinze dernières années sont à cet égard particulièrement marquants. Je citerai tout d'abord, en 1983, la création d'un Bureau des Musiques Traditionnelles à la Direction de la Musique et de la Danse (DMD) au Ministère de la Culture. Désormais, les acteurs du revival, de même que les ethnomusicologues du domaine français, vont disposer d'un interlocuteur officiel au niveau du Ministère de la Culture, notamment en la personne d'un Inspecteur principal chargé des musiques traditionnelles, poste occupé depuis sa création uniquement par des ethnomusicologues (MM. Bernard Lortat-Jacob, Michel De Lannoy, Jean-Pierre Estival). La primauté de l'ethnomusicologie dans un domaine vaste et complexe incluant aussi bien la pratique musicale et chorégraphique vivante que son espace (le domaine de la diffusion musicale), que la formation ou encore la documentation, me semble-t-il, est très révélatrice d'une imbrication profonde entre ethnomusicologie et action culturelle dans le secteur des musiques traditionnelles en France. Poursuivant son action de structuration et de dynamisation, le Ministère de la Culture, plus exactement la DMD, crée en 1985 la Fédération des Associations de Musiques Traditionnelles, devenue depuis Fédération des Associations de Musiques et Danses Traditionnelles (FAMDT). En 1987, la DMD offre aux musiciens traditionnels, pour la première fois, la possibilité d'accéder aux diplômes nationaux d'enseignement musical (Diplôme d'Etat, Certificat d'Aptitude aux fonctions de chef de département). La musique traditionnelle, déjà présente dans nombre de 
structures institutionnelles d'enseignement musical en Provence (Marseille, Aix-enProvence, Arles, Nice, etc.), va faire son entrée dans de nombreux Conservatoires et Ecoles de musique en France (Bayonne, Limoges, Pontivy, Ecole départementale de la Creuse, de la Vienne, du Lot, du Tarn, de l'Aveyron, de la Lozère, etc.). En 1989, sous l'égide de la FAMDT et de la DMD sont organisées à Paris (Maison des Cultures du Monde) les $2^{\mathrm{e}}$ Assises des Musiques Traditionnelles, au cours desquelles vont naître les prémices des futures commissions thématiques et transversales de la FAMDT (dans les domaines de la recherche, de la documentation, de la pratique musicale, de la danse) et au terme desquelles va se manifester la nécessité impérieuse de créer des réseaux et de les faire fonctionner. On peut voir véritablement dans ces Assises l'origine de la notion de «mise en réseau » constitutive des Centres de Musiques et Danses Traditionnelles en Régions que la DMD crée dès 1990 dans sept régions françaises, en s'appuyant la plupart du temps sur des associations déjà existantes : en Bretagne, c'est l'association Dastum qui devient Centre des Musiques Traditionnelles; en Poitou-Charentes, c'est l'UPCP ; en Aquitaine, le Centre Lapios; en Auvergne, l'Agence des Musiques Traditionnelles d'Auvergne ; en MidiPyrénées, le Conservatoire Occitan ; en Ile-de-France, la MJC de Ris-Orangis ; et en RhôneAlpes, un Centre est créé de toutes pièces : le Centre des Musiques Traditionnelles RhôneAlpes. Depuis, un Centre des Musiques Traditionnelles a vu le jour en Limousin, un autre en Languedoc-Roussillon, un autre en Provence-Alpes-Côte-d'Azur. Enfin, un dernier est à l'étude en Corse. Cette notion de "mise en réseau ", si caractéristique du nouveau découpage français du domaine des musiques traditionnelles, n'est pas exclusivement «franco-française »: elle doit prendre en compte la diversité culturelle et musicale des diverses communautés installées dans les régions, au moins celles qui sont dotées de Centres. Par exemple, dans le domaine de la diffusion (on appelle «diffusion» la présentation publique des pratiques musicales « vivantes " sous forme de concerts, bals, animations, et spectacles divers), le préambule qui lie les Centres des Musiques Traditionnelles à la DMD est très clair : « Dans le domaine de la diffusion, [les actions de mise en réseau] s'appliqueront à assurer la promotion du spectacle vivant, en organisant lui-même [le Centre] une programmation ou en s'associant à des initiatives régionales de programmation ou de création musicale ou chorégraphique: ces programmations intégreront l'éventail le plus large possible des musiques et danses traditionnelles de France et du monde ».

11 Structuration, dynamisation du domaine des musiques traditionnelles en France, mais aussi professionnalisation de leurs principaux acteurs, telle est la politique menée depuis treize ans maintenant par le Ministère de la Culture et relayée par les collectivités locales. Cette politique tend à " officialiser » un secteur par nature particulièrement jaloux de son autonomie, de même qu'elle tend à « officialiser » la réalité culturelle des communautés immigrées. Ce phénomène très récent est certainement l'un des faits les plus marquants de ces dernières années. Mais cette politique est à la fois délicate à mettre en œuvre face à des réflexes communautaires parfois méfiants, de même qu'elle peut présenter certains dangers intrinsèques, notamment celui d'une acculturation supplémentaire.

Dans le domaine de la recherche, on assiste à une volonté de formation universitaire chez un certain nombre de collecteurs, ces derniers optant généralement pour un diplôme ou un doctorat de l'Ecole Pratique des Hautes Etudes ou de l'Ecole des Hautes Etudes en Sciences Sociales. On assiste donc à l'émergence d'une ethnomusicologie revivaliste du domaine français dont la méthodologie est désormais beaucoup plus rigoureuse et scientifique. Très récemment, et sous l'impulsion notable de la Direction de la Musique et 
de la Danse au Ministère de la Culture (notamment de Jean-Pierre Estival, Inspecteur chargé des musiques traditionnelles, véritable trait d'union personnel et institutionnel entre l'ethnomusicologie actuelle du domaine français et celle des domaines extraeuropéens - il est lui-même américaniste), plusieurs ethnomusicologues du domaine français, par ailleurs acteurs culturels, ont fait leur entrée à la Société Française d'Ethnomusicologie. D'autre part, on assiste depuis 1990 à l'élargissement du champ d'étude de cette nouvelle ethnomusicologie du domaine français à une anthropologie musicale historique qui prend en compte également la dimension de la danse. Dans l'ensemble, on note une persistance d'une ethnomusicologie de proximité, de " voisinage » immédiat, puisque, dans la plupart des cas, l'ethnomusicologue du domaine français est l'un des acteurs de son propre «terrain». Par son action, et surtout par l'utilisation qu'il propose de sa recherche (la notion de restitution est primordiale avec, très souvent, une rapide publication écrite ou sonore), il participe à l'anti-acculturation. Son ethnomusicologie est, en majorité, une ethnomusicologie "de l'utile», une ethnomusicologie « militante ».

On voit donc à travers ce développement chronologique à quel point les histoires et les approches diffèrent entre les ethnomusicologues actuels du domaine français et ceux des domaines extra-français et extra-européens. Car à l'évidence, chez les premiers, l'antériorité culturelle sur l'approche scientifique, et même l'engagement culturel en parallèle à l'investigation scientifique, limitent considérablement la distanciation culturelle avec l'objet étudié. Dans le champ plus large de l'ethnomusicologie, nombreuses sont les situations d'une relative « extériorité » de l'ethnomusicologue face à son « terrain ». L'histoire même de l'ethnomusicologie en est à l'origine. Cette discipline, longtemps exclusivement occidentale, en était arrivée à définir son propre terrain: les traditions musicales les plus éloignées culturellement de la sphère occidentale. Seule l'étude des traditions musicales "primitives» était censée pouvoir apporter une explication rationnelle à la musicologie quant à la formation de la musique, à sa pratique "pré-historique ", au mythe des origines. On ne retenait alors de l'action d'un Brâiloiu que sa méthode et non l'objet de ses investigations : la ruralité d'une région européenne. Si bien que l'ethnomusicologie - tout comme l'ethnologie d'ailleurs dont le cheminement est parallèle - porta sur des rivages lointains des scientifiques tout à la fois observateurs et analystes, dont la fonction en faisait des médiateurs obligés entre les ethnies étudiées et le reste du monde, parfois réellement engagés (comme certains ethnologues) contre des processus d'acculturation, d'asservissement politique, culturel ou racial (Hugo Zemp n'aurait pas pu relater sa recherche sur les Iles Salomon sans y mentionner la dimension du combat politique des Mélanésiens pour leur indépendance). Quelles que soient les intentions de ces ethnomusicologues, leur distanciation avec leur terrain est posée comme un élément constitutif de leur démarche. Qu'advient-il de cette distanciation chez des ethnomusicologues européens travaillant sur des domaines européens? Est-elle, de fait, moins importante? Rien n'est moins sûr. Chez la plupart d'entre eux, le rapport aux faits musicaux étudiés et aux groupes sociaux qui en sont porteurs n'est pas fondamentalement différent de celui qui a cours chez les ethnomusicologues travaillant sur des domaines culturellement éloignés. La distance culturelle avec la ruralité, parfois la distance sociale aussi, la distance que s'impose le scientifique, produisent des situations analogues: en quoi le rapport de Claudie Marcel-Dubois avec les paysans et bergers de l'Aubrac, des Landes ou des Pyrénées est-il fondamentalement différent de celui d'un américaniste, d'un africaniste ou d'un océaniste avec les hommes et femmes de son terrain? Il n'est évidemment pas question ici de porter le moindre jugement de valeur 
sur toutes ces approches, si nombreuses, si diverses et si fécondes. Mais, à l'évidence, les enjeux ne sont pas les mêmes avec la plupart des ethnomusicologues actuels du domaine français, même si, chez ces derniers, la question du rapport au terrain, c'est-à-dire finalement la question de l'identité, mériterait d'être posée et débattue. Cette proximité culturelle et affective induit une approche tout autre, et le plus souvent un engagement très «militant». Cette démarche n'est d'ailleurs pas exclusive au domaine "francofrançais »: un Marc Loopuyt, dont les attaches personnelles avec certains pays du Maghreb sont très fortes, qui découvre la musique à travers l'apprentissage de la guitare flamenca auprès d'un de ses voisins espagnol résidant dans la région strasbourgeoise, qui part étudier auprès des plus grands maîtres proche-orientaux et qui, au bout du compte, dispense dans la région lyonnaise une formation en musique andalouse et procheorientale pour un public majoritairement immigré, s'inscrit totalement dans une démarche ethnomusicologique militante.

Fig. 3 : Didier Champion et Yvan Karveix, musiciens « revivalistes » auvergnats, font danser le public des journées de la Danse Traditionnelle organisées par le Conservatoire Occitan et la ville de Colomiers (Haute-Garonne). 1994. Collection Conservatoire Occitan.

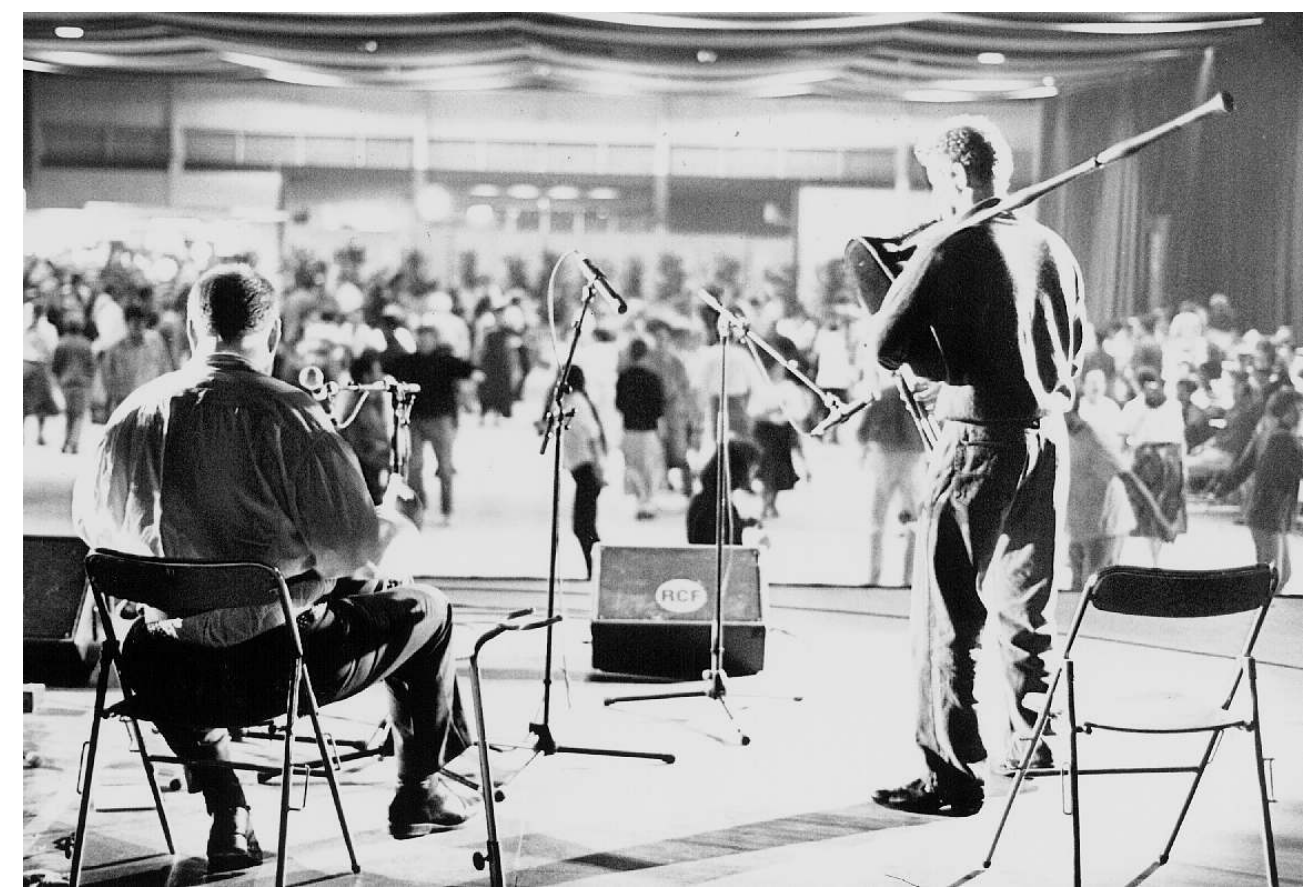

Lorsque l'on évoque l'émergence d'une ethnomusicologie revivaliste du domaine français, il convient cependant d'en marquer les limites: les ethnomusicologues sont encore peu nombreux, de même que l'utilisation qui est faite de leur recherche est, dans le paysage musical traditionnel français actuel, assez peu importante $e^{4}$. Entre les tenants du courant folk (rejet de toute démarche qui pourrait être perçue comme une marque de "purisme »; référence à des influences multiples sans distinction) et les musicalistes (la musique traditionnelle est vécue et pratiquée comme n'importe quel autre genre musical, sans connotation idéologique particulière) dans lesquels il faut peut-être inclure les tenants de la world-music, du métissage (à moins que cette quête de « fusion » ne procède d'un projet culturel), il reste peu d'espace musical concerné par le ressourcement proposé par l'ethnomusicologie. Non pas que ces expressions musicales négligent totalement toute référence aux sources de la musique, du chant ou de la danse; mais elles ne les intègrent pas dans un projet. Les seuls, peut-être, à envisager une utilisation consciente et 
raisonnée des documents ethnomusicologiques se situent chez les régionalistes. Mais alors de quelle ethnomusicologie s'agit-il ? Ne prend-on pas le risque de mythifier les résultats, de proposer l'établissement de modèles, de normes qui vont parfois jusqu'à travestir la réalité ? Ceci dit, l'intérêt que portent les régionalistes aux données fondamentales de l'ethnomusicologie se retrouve dans la typologie des prestations musicales. Alors que les musiciens folk, les musicalistes, les partisans de la world-music revêtent une pratique essentiellement scénique (concert ou bal traditionnel), les régionalistes, en plus du bal ou $\mathrm{du}$ concert, ont une action beaucoup plus circonstancielle, sociale et communautaire (fêtes calendaires et traditionnelles - bœufs-gras, joutes nautiques, carnavals, etc. -, fêtes de proximité - veillées...-, vie du quartier, de la ville, etc.). Le Conservatoire Occitan, par exemple, indépendamment de toute considération politique, est engagé assez nettement dans ce processus musical. Cela se lit aussi dans le soutien puissant et indéfectible de la Ville de Toulouse.

qu'il serait prétentieux d'exagérer la portée de l'ethnomusicologie du domaine français, il serait également faux de la présenter comme consensuelle. Car, outre la notion d'identité - si prégnante dans la typologie énoncée ci-dessus -, celles de tradition et surtout de sociétés traditionnelles et de leur possible survivance sont également l'objet d'un vif débat. D'un côté, pour résumer à l'extrême - ce débat a principalement cours, actuellement, dans le domaine de la danse traditionnelle -, il y a ceux qui postulent une rupture du type de pratique et de transmission de la danse traditionnelle, due à la disparition définitive des sociétés traditionnelles paysannes françaises. Dans ce cas, tout ce qui est aujourd'hui tangible (les acteurs du mouvement de renouveau des musiques traditionnelles comme les témoins qu'ils ont collectés) ne serait que revivalisme. Et puis, à l'inverse, il y a ceux qui estiment que certaines pratiques ont survécu au déclin par ailleurs général. Dans ces cas-là, ils dénient la notion de revival puisque, selon eux, il n'y a pas eu rupture. Le mouvement actuel en faveur des musiques et danses traditionnelles n'est alors que la poursuite de phénomènes plus anciens, même s'il y a évolution et adaptation. Voilà donc, grosso modo, les deux thèses en présence.

La première, qui a trouvé deux ardents défenseurs chez Jean-Michel et Yves Guilcher - et dans leurs travaux sur la danse traditionnelle comme révélateur des sociétés traditionnelles (Guilcher J.-M. 1971 : 7-49; Guilcher Y. $1992:$ 8-26) -, reprend finalement les thèses de la plupart des historiens de la paysannerie française, de Mendras à Eugène Weber: dès le début de l'ère industrielle, on assiste à la disparition des sociétés traditionnelles paysannes. Ces micro-sociétés, qui fonctionnaient alors comme des "sociétés d'inter-connaissance ", c'est-à-dire vivant dans une grande autarcie et sans grandes influences extérieures notables, étaient elles-mêmes productrices de faits musicaux et chorégraphiques traditionnels. Mais l'industrialisation, génératrice de communication à grande échelle et d'une internationalisation de l'économie aurait irrémédiablement détruit ce schéma. S'appuyant sur l'exemple de la Bretagne, qui lui est si cher, Jean-Michel Guilcher montre ensuite en quoi ce que l'on aurait pu être fondé à interpréter comme des marques de survivances de pratiques chorégraphiques traditionnelles ne sont en fait que les effets d'une reconstruction de type idéologique (en particulier due au folklore, avant même le revival). A cela, on peut avancer un certain nombre de faits susceptibles de relativiser cette hypothèse. Tout d'abord, peut-on parler de sociétés "d'inter-connaissance » alors que, dans le domaine qui nous préoccupe, la circulation des musiciens, des musiques et des danses est générale en France depuis la Renaissance, dans les villes mais aussi très certainement dans des endroits plus reculés, et 
que cette circulation des répertoires et des individus s'est opérée dans le cadre d'une organisation structurée à l'échelon du royaume et centralisée, la Ménestrandise (CharlesDominique 1994) ? (Cette question, que je me suis permis de poser à Jean-Michel Guilcher récemment, reste pour l'instant sans réponse). D'autre part, on pourrait trouver une quantité de contre-exemples fameux d'échanges directs entre les sociétés paysannes et les sociétés urbaines, qui ont de tout temps exercé une véritable fascination sur les ruraux. Cela pose la question - mais l'étude pourrait être étendue à l'économie tout entière, à l'évolution du " goût ", des techniques, etc. - de la pertinence d'un modèle de société monolithique qui n'aurait subi que peu d'apports extérieurs jusqu'au début du xix e siècle, et surtout de l'unicité de ce modèle de société, ne serait-ce que dans le cadre si complexe, si varié, si mal connu encore - de ce point de vue-là - de la France.

Quant à la seconde thèse en présence, les tenants d'une transmission sans rupture ne peuvent nier les profondes mutations qu'ont connues les musiciens - ou les danseurs qui ont pu être nos informateurs vers les années 1970 et qui ont exercé dans les années 1920-1930. Ignorer les échanges, les influences, l'évolution des contextes, l'apport éventuel de l'écrit ou d'une formation autre que celle de l'imprégnation, etc., reviendrait à occulter des données essentielles à la compréhension des phénomènes ethnomusicologiques et ethnochoréologiques.

Au-delà des excès que revêt cette polémique et dont l'acuité a peut-être des raisons purement existentielles (imagine-t-on la portée de la thèse des partisans d'une rupture totale et définitive des traditions musicales et chorégraphiques, qui annule d'un seul coup les conclusions de travaux ethnomusicologiques sur le domaine français de ces dix à quinze dernières années ?), il semble que, bien plus que la notion d'identité, celle de tradition soit actuellement au centre des polémiques et des débats ${ }^{6}$. Sans doute parce que, au-delà du flou intrinsèque à cette notion, l'interprétation rationnelle des faits ethnomusicologiques est rendue très complexe en raison même de l'inaptitude que l'on éprouve à évaluer et à comprendre une société dans sa globalité, à percevoir les mécanismes de son évolution ou de son déclin. Jusqu'à quel point sommes nous fondés, dans un domaine scientifique aussi peu exact que celui de l'ethnologie - et donc de l'ethnomusicologie -, à quantifier et à pronostiquer les effets de l'acculturation?

\section{BIBLIOGRAPHIE}

BOILES Charles, NATTIEZ Jean-Jacques, 1977, « Petite histoire critique de l'ethnomusicologie », Musique en jeu 28 : 26-53.

CHARLES-DOMINIQUE Luc, 1994, Les Ménétriers français sous l'Ancien Régime, Paris, Klincksieck.

ESTIVAL Jean-Pierre,, 1995, « Musiques traditionnelles : une approche du paysage français » Internationale de l'Imaginaire, nouvelle série, $4:$ « La Musique et le Monde », Paris, Maison des Cultures du Monde : 147-157.

GUILCHER Jean-Michel, 1971, « Aspects et problèmes de la danse populaire traditionnelle », Ethnologie française, Nouvelle série, Tome 1, $\mathrm{N}^{\circ} 2$ : 7-49. 
GUILCHER Yves, 1992, «La danse renseigne sur plus qu'elle-même », Danse et Société, Actes du colloque (Toulouse, 29 octobre 1988), Toulouse, Conservatoire Occitan : 8-26.

ZEMP Hugo, 1978, Polyphonies des Iles Salomon (Guadalcanal et Savo), 33 tours Le Chant du Monde, collection CNRS/ Musée de l'Homme LDX 74663, accompagné d'un livret bilingue françaisanglais.

\section{NOTES}

1. Je conçois parfaitement que tous les ethnomusicologues actuels du domaine français ne se reconnaissent pas dans ce type d'ethnomusicologie, ou en tout cas dans ses objectifs. Le propos de cet article est de montrer, néanmoins, la réalité actuelle d'une telle forme d'ethnomusicologie, et à travers son exposition schématique, de rappeler sa composante identitaire qui reste, à mon avis dans le cas présent, essentielle et fondatrice.

2. Afin d'éviter toute confusion dans la suite de mon propos, je différencierai nettement l'ethnomusicologie du domaine français inaugurée et poursuivie durant plusieurs décennies par Claudie Marcel-Dubois et Maguy Andral dans le cadre du Musée National des Arts et Traditions Populaires et de plusieurs instances universitaires et scientifiques, de celle beaucoup plus tardive élaborée dans le cadre du mouvement (associatif) de renouveau des musiques traditionnelles. Dans la suite de l'article, lorsqu'il sera question de l'ethnomusicologie du domaine français, c'est à cette dernière que je ferai allusion, et afin d'éviter toute confusion, je la qualifierai le plus possible «d'actuelle». D'autre part, par «ethnomusicologie du domaine français », j'entends l'ethnomusicologie rurale et urbaine de la France à l'exception de celle des communautés immigrées, dont l'approche - et la situation générale - est très différente.

3. L'intitulé de l'association, dans les statuts de 1970, est Conservatoire Occitan des Arts et Traditions Populaires.

4. La typologie qui suit est extrêmement schématique, très peu nuancée et forcément caricaturale. Mais son développement nous entraînerait très loin et dépasserait sans doute les limites de cette contribution. Néanmoins, l'opposition musicalistes-régionalistes fut réellement posée comme telle lors d'une session de réflexion de la Fédération des Associations de Musiques et Danses Traditionnelles (FAMDT), organisée les 11 et 12 janvier 1990 à L'Isle-Jourdain (Gers) sur le thème de l'ancrage identitaire ou non de la musique traditionnelle ou, dans ce cas-là, de son inscription dans l'éventail des divers courants esthétiques musicaux. Le mot « régionaliste "ne revêt pas ici de connotation politique particulière. Il est plutôt synonyme d'identité, une identité «mythique ou réelle [...] qui peut être liée à une ville, un pays, une région, quelques individus... avec une tension paradoxale entre l'identité individuelle et l'identité de groupe... ». Soucieuse de parvenir à une synthèse, la FAMDT publiait en 1992 un « manifeste » dans lequel elle affirmait sa «volonté de résistance à la mono-culturisation mondiale», tout en revendiquant «le droit de chacun à l'autonomie de la mémoire musicale [...], la liberté de l'expression musicale [...], le droit de chacun à s'épanouir dans la diversité de ses identités... ». De la sorte, elle considérait sa démarche comme «transculturaliste et universaliste ».

5. Le concept est de Jean-Michel Guilcher. (Conférence d'introduction aux Journées d'étude de la Société Française d'Ethnomusicologie, Saint-Malo, novembre 1995).

6. Un des exemples les plus récents en est les Journées d'Etude de la Société Française d'Ethnomusicologie de Saint-Malo (novembre 1995) consacrées au thème de la tradition. 


\section{RÉSUMÉS}

Face aux phénomènes d'acculturation et d'hybridation musicales que l'ethnomusicologue constate à l'échelon de la planète tout entière, il est amené la plupart du temps à reconsidérer son objet, ses méthodes et aussi à se poser la question de son engagement personnel. En Europe, et plus particulièrement en France, le problème se pose un peu différemment, d'une part en raison de la précocité avec laquelle ces phénomènes sont apparus, d'autre part en raison d'une histoire culturelle et politique particulière (le rejet des cultures « dominantes ", la revendication régionaliste et écologiste), qui est à l'origine d'un engagement culturel avant même que le champ ainsi élaboré des musiques traditionnelles ne connaisse une approche plus scientifique.

Cette ethnomusicologie revivaliste du domaine français, qui n'émergera que dans le courant de la décennie 1980, se développera parallèlement à la maturation, à la structuration et à la professionnalisation du secteur des musiques traditionnelles dans lesquelles la constance de la politique du Ministère de la Culture, depuis 1983, joue tout son rôle. Cette ethnomusicologie, dont la portée n'est encore que relative, se pose souvent comme une ethnomusicologie «de l'utile », comme une ethnomusicologie prospective et militante, avec en arrière-plan des débats fondamentaux qui la divisent sur la revendication régionaliste et identitaire ou sur la notion de tradition, plus exactement sur la perpétuation ou non des sociétés traditionnelles et sur la survivance ou non de pratiques musicales et chorégraphiques traditionnelles.

When faced with the musical multi-culturization and hybridity that the ethnomusicologist encounters over the whole planet, he is led, in most cases, to reassess the 'object' of his study, his methods and also to ask some pertinent questions regarding his own involvement. In Europe and more particularly in France, the question presents itself slightly differently. On the one hand because of the early stage at which these phenomena appeared and on the other, because of special political and cultural history i.e. the rejection of the notion of dominant cultures and regional and ecological demands, was at the origin of cultural commitment even before the field of traditional music thus elaborated adopted a more scientific approach.

This type of revivalist French ethnomusicology, which only came to light in the mid nineteen eighties, was to develop in parallel with the coming of age, the structuring and professionalism of the traditional music sector, in which the constancy of the Ministry of Culture, from 1983 on, played a full part. This ethnomusicology, the influence of which is for the time being quite relative, often presents itself as 'practical ethnomusicology', as ethnomusicology which is involved and active, with, in the background fundamental debates which divide it on matters such as regional demands, identity or on the notion of what is meant by tradition. To be more precise, on points such as the perpetuation or not of traditional societies and on the survival or not of musical practices and traditional choreography.

\section{AUTEUR}

\section{LUC CHARLES-DOMINIQUE}

Luc Charles-Dominique, né en 1955, entre en 1977 comme musicien et chercheur au Conservatoire Occitan de Toulouse, dont il est depuis 1985 le directeur musical. Concepteur et 
directeur musical de la collection discographique anthologique « Musiques et voix traditionnelles aujourd'hui », rédacteur en chef de la revue de musique traditionnelle Pastel, auteur de plusieurs ouvrages sur la musique traditionnelle occitane, il mène depuis de nombreuses années une recherche plus spécifique en anthropologie musicale historique, tout d'abord sur l'histoire de la corporation des ménétriers de Toulouse (Ecole des Hautes Etudes en Sciences Sociales), puis plus largement sur Les Ménétriers français sous l'Ancien Régime (ouvrage qu'il publie chez Klincksieck en 1994), et sur la symbolique sociale et religieuse des instruments de musique en France, du Moyen-Age à la fin de l'Ancien Régime. Il est par ailleurs chargé de cours en ethnomusicologie dans les Universités de Toulouse-le-Mirail et de Nice-Sophia-Antipolis. 